\title{
Treatment outcomes among patients admitted to hospital with antiretroviral and/or antituberculosis drug-induced liver injury
}

R Mehta, ${ }^{1}$ MB ChB, FCP (SA), MMed (Int Med); P Ive, ${ }^{2}$ MB BCh, FCP (SA), DTM\&H, Dip HIV Man (SA), Cert ID (SA) Phys; D Evans, ${ }^{3}$ DBiomed; C N Menezes, ${ }^{4} \mathrm{MD}$, MMed (Int Med), Dip HIV Man (SA), DTM\&H, FCP (SA), Cert ID (SA), PhD

\author{
${ }^{1}$ Department of Internal Medicine, School of Clinical Medicine, Faculty of Health Sciences, University of the Witwatersrand, Johannesburg, \\ South Africa \\ ${ }^{2}$ Division of Infectious Diseases, Helen Joseph Hospital and Department of Internal Medicine, School of Clinical Medicine, Faculty of Health \\ Sciences, University of the Witwatersrand, Johannesburg, South Africa \\ ${ }^{3}$ Health Economics and Epidemiology Research Office, Department of Internal Medicine, School of Clinical Medicine, Faculty of Health Sciences, \\ University of the Witwatersrand, Johannesburg, South Africa \\ ${ }^{4}$ Division of Infectious Diseases, Chris Hani Baragwanath Hospital and Department of Internal Medicine, School of Clinical Medicine, \\ Faculty of Health Sciences, University of the Witwatersrand, Johannesburg, South Africa
}

Corresponding author: R Mehta (ruchikasmehta@gmail.com)

\begin{abstract}
Background. South Africa (SA) has among the highest rates of HIV and tuberculosis (TB) in the world. Antituberculosis and antiretroviral treatment (ART) can cause drug-induced liver injury (DILI), consequences of which are disease relapse, treatment failure and drug resistance. Objectives. To: (i) determine the demographics of patients with DILI and the proportion of patients on antituberculosis drugs v. antiretroviral therapy or both; (ii) determine the median time to DILI after starting medication, and patterns of clinical presentation; (iii) determine the numbers of patients successfully re-challenged to initial therapy as inpatients; and (iv) determine the in-hospital mortality rate and predictors of all-cause mortality.

Methods. This was a retrospective record review of adult patients with DILI admitted to a tertiary hospital in Johannesburg, SA, between October 2015 and February 2017. Data on drug history, biochemical investigations and relevant imaging were collected.

Results. The total sample was 129 records: 79 (61.2\%) were males, 46 (35.7\%) had TB DILI, 29 (22.5\%) had ART DILI, and 54 (41.9\%) had mixed TB/ART DILI. Only 7.4\% (2/27) of those with ART DILI and 30.6\% (11/36) with TB DILI were re-challenged to their original regimen by discharge. Patients were followed from admission until the earlier of death (10 with TB DILI, 2 with ART DILI and 9 with mixed DILI) or discharge (after a median (interquartile range) of 14.0 (9 - 23) days). In adjusted analysis, severe DILI at admission predicted allcause mortality (adjusted hazard ratio 8.58 ; 95\% confidence interval 1.13 - 65.4).

Conclusions. This study is one of only a few analyses of hospitalised patients with DILI in SA. Among those with severe DILI, outcomes are poor, the majority cannot tolerate standard regimens, and mortality is high.
\end{abstract}

S Afr Med J 2021;111(5):474-481. https://doi.org/10.7196/SAMJ.2021.v111i5.15353

South Africa (SA) has one of the highest prevalence rates of HIV in the world. ${ }^{[1]}$ In 2019, the prevalence of HIV in SA was $19.07 \%$ among adults aged 15 - 49 years; $25.8 \%$ among females and $15 \%$ among males. ${ }^{[1]}$ Tuberculosis (TB), the most common opportunistic infection affecting HIV-positive individuals, continues to be the leading cause of death in SA. ${ }^{[1]}$

Although the benefits of antiretroviral treatment (ART) and antiTB treatment have been clearly demonstrated, both are associated with adverse drug reactions (ADRs). In general, HIV patients have a higher rate of $\mathrm{ADRs}$ to both $\mathrm{TB}$ and non-TB medications than the general population, and the risk of ADRs increases with the degree of immunosuppression. ${ }^{[2]}$ Drug-induced liver injury (DILI) is a well-recognised ADR of TB treatment and ART. It is estimated that DILI affects 5 - 33\% of patients on TB treatment, although local data are limited ${ }^{[3]}$ Depending on the regimen, $9-30 \%$ of patients on ART develop DILI. ${ }^{[4]}$ DILI complicates therapy, and can potentially affect adherence, lead to interruption of treatment and contribute to unfavourable outcomes. ${ }^{[5,6]}$ TB DILI in HIV co-infection is the most common adverse effect of treatment necessitating drug interruption, ${ }^{[5]}$ which can lead to treatment failure, relapse or drug resistance. ${ }^{[6]}$ Inpatient mortality associated with hepatoxicity from anti-TB medication is high (27\%). ${ }^{[6]}$

Concurrent treatment for HIV and TB creates several management challenges. ${ }^{[7]}$ Because ADRs are among the most important factors resulting in interruption of ART and anti-TB treatment, monitoring and managing adverse reactions is important.

\section{Objectives}

Local data on DILI are limited, so this study aimed to describe the characteristics and outcomes of patients with DILI presenting to a tertiary hospital in Johannesburg, SA. The results may help inform guidelines on the management of DILI, thereby improving treatment outcomes among patients on treatment for HIV and/or TB.

\section{Methods}

We conducted a retrospective review of an electronic database of HIV-positive adult ( $\geq 18$ years) patients with DILI admitted to Helen Joseph Hospital, a 500-bed tertiary academic hospital in Johannes- 
burg, between October 2015 and February 2017. Johannesburg is an area with a high burden of TB-HIV coinfection.

TB DILI is defined by the Southern African HIV Clinicians Society (SAHIVCS) as elevated alanine transaminase (ALT), >120 IU/L in symptomatic patients (nausea, vomiting, malaise, loss of appetite, abdominal pain or jaundice) or $>200 \mathrm{IU} / \mathrm{L}$ in asymptomatic patients, or total serum bilirubin $>40 \mathrm{mmol} / \mathrm{L}$, provided other aetiologies such as acute viral hepatitis, autoimmune hepatitis and other liver diseases are ruled out. ${ }^{[3]}$ The upper limit of normal for ALT and total bilirubin according to the SA National Health Laboratory Service reference range is $40 \mathrm{IU} / \mathrm{L}$ and $21 \mathrm{mmol} / \mathrm{L}$, respectively. Patients are managed according to the SAHIVCS guideline. ${ }^{[3]}$

\section{Materials}

Patient information on demographics, presenting symptoms, date of onset of drug therapy, drug regimen (including other hepatotoxic drugs) and diagnosis of TB was recorded in an electronic database.

Liver function tests (alkaline phosphatase (ALP), gamma-glutamyl transferase, total bilirubin, ALT, aspartate aminotransferase and international normalised ratio) were monitored on admission and serially as indicated. Results of other tests, including CD4 count, HIV viral load, hepatitis $\mathrm{B}$ and hepatitis $\mathrm{C}$ status, autoimmune work-up, abdominal ultrasound and liver biopsy (if done to rule out other causes of liver injury), were collected.

\section{Measures}

TB patients were classified as bacteriologically confirmed if they were diagnosed through Xpert MTB/RIF (Cepheid, USA), smear microscopy or culture. Patients diagnosed via X-ray were classified as clinically diagnosed, while those with missing diagnostic information who were on anti-TB medication were considered to have presumptive $\mathrm{TB} .{ }^{[8]}$

TB DILI was defined as DILI in a patient with TB/HIV who was exclusively on anti-TB therapy, ART DILI as DILI occurring in a patient only on ART, and mixed DILI as occurring in a patient on both ART and anti-TB therapy.

The liver function tests were used to derive the $\mathrm{R}$ value (patient's ALT/40 divided by ALP/120), which was used to classify the pattern of liver enzyme elevation as hepatocellular $(R \geq 5)$, cholestatic $(R \leq 2)$ or mixed $(\mathrm{R}>2$ but $<5)$.

Based on the SAHIVCS definition, the patient's symptoms and liver enzymes were used to categorise the severity of DILI as mild (patient clinically well, with ALT $>120$ but $<200$ IU/L, total bilirubin $<40 \mathrm{mmol} / \mathrm{L}$ ), moderate (patient clinically well, with ALT $>200 \mathrm{IU} / \mathrm{L}$ regardless of bilirubin results) or severe (patient clinically unwell and meets the DILI criteria).

The ART and anti-TB drug regimen on discharge was documented to establish how many patients were successfully re-challenged back to the initial drug regimen.

\section{Statistical analysis}

Demographic and clinical characteristics were summarised using frequencies for categorical variables and means with standard deviations for normally distributed data or medians and interquartile ranges (IQRs) for data not normally distributed. To compare demographic and clinical characteristics at baseline between the different types of DILI, we used the Wilcoxon rank-sum or KruskalWallis test for non-parametric data, Student's $t$-test for parametric or normally distributed data, and the $\chi^{2}$ test (or Fisher's exact test for sparse data) for proportions.

The primary outcome was all-cause mortality, predictors of which were assessed using Cox proportional hazards regression. Patients were followed from date of admission until the earliest of death or discharge. Variables with a $p$-value $<0.25$ in the univariate analysis along with a priori variables were included in the final multivariate model. The hazard ratio (HR) and corresponding 95\% confidence interval (CI) are presented. Because the number with the outcome of interest was limited $(n=21)$ and to minimise the risk of overfitting, the final model was restricted by the number of predictors that could be added.

We determined whether use of concomitant medication (e.g. fluconazole, co-trimoxazole, amoxicillin-clavulanic acid, paracetamol or herbal medication) or abnormal ultrasound results (e.g. enlarged liver, echogenic, liver mass, micro-abscesses, intra-abdominal nodes or ascites) were associated with presenting with severe DILI. We used a log-binomial regression model to estimate the crude relative risk (RR) and 95\% CI.

Sensitivity analysis considered all-cause mortality within 60 days, and patients were followed from the date of admission until the earliest of death, discharge or 60 days in hospital care. We used Kaplan-Meier survival curves to display survival probabilities from hospital admission within 60 days of follow-up for all-cause mortality. Analyses were conducted in Statistica and SAS version 9.3 (SAS Institute, USA).

\section{Ethical considerations}

Ethics approval was granted by the Human Research Ethics Committee (Medical) of the University of the Witwatersrand, Johannesburg (ref. no. M170324).

\section{Results}

A total of 129 patients were included ( $n=79$ (61.2\%) males); the median (IQR) age was 35.4 (31.4 - 43.5) years, and $46(35.7 \%)$ had TB DILI, 29 (22.5\%) ART DILI and 54 (41.9\%) mixed DILI (TB and ART).

Six patients were hepatitis B surface antigen-positive, but no patients were hepatitis $\mathrm{C}$ antibody-positive. Sixteen patients had an autoimmune screen; 3 were positive for antinuclear factor, whereas all antimicrosomal antibodies and anti-liver-kidney antibodies were negative (Table 1).

The commonest symptom on presentation was nausea and/or vomiting ( $n=60$ patients; $46.5 \%$ ) while $55(42.6 \%)$ had jaundice and $24(18.6 \%)$ abdominal pain. Jaundice was more common in patients with ART DILI (72.4\%) than in those with TB DILI (23.9\%) and mixed DILI $(42.6 \%)(p<0.05)$.

\section{Patients on ART}

On admission, 83 HIV-positive patients were on ART; $89.2 \%$ of them were on a fixed-dose combination (FDC) of tenofovir/emtricitabine/ efavirenz. Two-thirds $(n=54 / 83 ; 65.1 \%)$ presented with a mixed DILI and the remainder with ART DILI, consistent with the co-infection rates for Johannesburg. ${ }^{[8]}$ The median (IQR) CD4 count was 549 (345 - 741) cells/ $\mu \mathrm{L}$ in those with ART DILI, and $92.3 \%$ of patients had a viral load $<1000$ copies $/ \mathrm{mL}$. At presentation, most patients with mixed DILI were immunosuppressed $(74.1 \%$ with a CD4 count $<100$ cells $/ \mu \mathrm{L})$, had a detectable viral load $(54.3 \%$ had a viral load $\geq 100$ copies $/ \mathrm{mL}$ ), and were receiving concomitant hepatotoxic medication $(27.8 \%$ were on co-trimoxazole). The median time between ART initiation and DILI was 85 days in those with ART DILI v. 168 days in those with mixed DILI.

In two-thirds $(62.1 \%)$ of patients with ART DILI, DILI was classified as severe, whereas the figure dropped to $55.6 \%$ among those with mixed DILI. The median (IQR) ALT, total bilirubin and GGT were highest in the ART DILI group at 626 (292 - 1293$)$ IU/L, 


\begin{tabular}{|c|c|c|c|c|c|}
\hline & All $(N=129)$ & $\begin{array}{l}\text { TB DILI } \\
(N=46)^{\dagger}\end{array}$ & $\begin{array}{l}\text { ART DILI } \\
(N=29)^{*}\end{array}$ & $\begin{array}{l}\text { Mixed DILI } \\
(N=54)^{\varsigma}\end{array}$ & $p$-value \\
\hline \multicolumn{6}{|l|}{ Age (years) } \\
\hline Median (IQR) & $35.4(31.4-43.5)$ & $36.2(33.2-43.8)$ & $35.1(28.5-40.3)$ & $35.4(31.2-43.6)$ & 0.491 \\
\hline$<35, n / N(\%)$ & $58 / 129(45.0)$ & $19 / 46(41.3)$ & $14 / 29(48.3)$ & $25 / 54(46.3)$ & 0.812 \\
\hline$\geq 35, n / N(\%)$ & $71 / 129(55.0)$ & $27 / 46(58.7)$ & $15 / 29(51.7)$ & $29 / 54(53.7)$ & \\
\hline \multicolumn{6}{|l|}{ Gender, $n / N(\%)$} \\
\hline Female & $50 / 129(38.8)$ & $18 / 46(39.1)$ & $8 / 29(27.6)$ & $24 / 54(44.4)$ & 0.946 \\
\hline Male & $79 / 129(61.2)$ & $28 / 46(60.9)$ & $21 / 29(72.4)$ & $30 / 54(55.6)$ & \\
\hline \multicolumn{6}{|l|}{ Concomitant medication, $n / N(\%)$} \\
\hline Bactrim & $25 / 123(20.3)$ & $9 / 40(22.5)$ & $1 / 29(3.5)$ & $15 / 54(27.8)$ & $<0.05$ \\
\hline Fluconazole & $9 / 123(7.3)$ & $3 / 40(7.5)$ & $1 / 29(3.5)$ & $5 / 54(9.3)$ & 0.624 \\
\hline Paracetamol & $1 / 123(0.8)$ & $1 / 40(2.5)$ & $0 / 29(0)$ & $0 / 54(0)$ & 0.351 \\
\hline Amox-clavulanate & $12 / 123(9.8)$ & $4 / 40(10.0)$ & $0 / 29(0)$ & $8 / 54(14.8)$ & 0.095 \\
\hline \multicolumn{6}{|l|}{ Symptoms at presentation, $n / N(\%)$} \\
\hline Nausea and/or vomiting & $60 / 129(46.5)$ & $22 / 46(47.8)$ & $12 / 29(41.4)$ & $26 / 54(48.2)$ & 0.820 \\
\hline Abdominal pain & 24/129 (18.6) & $8 / 46(17.4)$ & $7 / 29(24.1)$ & 9/54 (16.7) & 0.682 \\
\hline Jaundice & $55 / 129(42.6)$ & $11 / 46(23.9)$ & $21 / 29(72.4)$ & $23 / 54(42.6)$ & $<0.05^{*}$ \\
\hline \multicolumn{6}{|l|}{ ART regimen at presentation, $n / N(\%)$} \\
\hline TDF/3TC/EFV & $74 / 83(89.2)$ & - & $26 / 29(89.7)$ & $48 / 54(88.9)$ & 0.573 \\
\hline TDF/3TC/NVP & $1 / 83(1.2)$ & - & $1 / 29(3.5)$ & $0 / 54(0)$ & \\
\hline $\mathrm{d} 4 \mathrm{~T} / 3 \mathrm{TC} / \mathrm{EFV}$ & $1 / 83(1.2)$ & - & $1 / 29(3.5)$ & $0 / 54(0)$ & \\
\hline AZT/3TC/EFV & $1 / 83(1.2)$ & - & $0 / 29(0)$ & $1 / 54(1.9)$ & \\
\hline $\mathrm{ABC} / 3 \mathrm{TC} / \mathrm{EFV}$ & $2 / 83(2.4)$ & - & $1 / 29(3.5)$ & $1 / 54(1.9)$ & \\
\hline $\mathrm{EFV} / 3 \mathrm{TC}$ & $1 / 83(1.2)$ & - & $0 / 0(0)$ & $1 / 54(1.9)$ & \\
\hline $\mathrm{ABC} / 3 \mathrm{TC} / \mathrm{LPVr}$ & $2 / 83(2.4)$ & - & $0 / 0(0)$ & $2 / 54(3.7)$ & \\
\hline $\mathrm{TDF} / 3 \mathrm{TC} / \mathrm{LPVr}$ & $1 / 83(1.2)$ & - & $0 / 0(0)$ & $1 / 54(1.9)$ & \\
\hline \multicolumn{6}{|l|}{ CD4 count, cells $/ \mu \mathrm{L}$} \\
\hline Median (IQR) & $66(20-228)$ & $37.5(13.0-70.0)$ & $549.0(345-741.0)$ & $56.0(19.0-108.0)$ & $<0.05^{*}$ \\
\hline$\leq 50, n / N(\%)$ & $56 / 129(43.4)$ & $28 / 46(60.8)$ & $2 / 29(6.9)$ & $26 / 54(48.2)$ & $<0.05^{*}$ \\
\hline $51-100, n / N(\%)$ & 24/129 (18.6) & 9/46 (19.6) & $1 / 29(3.5)$ & $14 / 54(25.9)$ & \\
\hline $101-250, n / N(\%)$ & $18 / 129(13.9)$ & $7 / 46(15.2)$ & $2 / 29(6.9)$ & $9 / 54(16.7)$ & \\
\hline $251-500, n / N(\%)$ & $12 / 129(9.3)$ & $2 / 46(4.4)$ & $7 / 29(24.1)$ & $3 / 54(5.5)$ & \\
\hline$>500, n / N(\%)$ & $19 / 129(14.7)$ & $0 / 46(0)$ & $17 / 29(58.6)$ & $2 / 54(3.7)$ & \\
\hline \multicolumn{6}{|l|}{ Viral load (copies/mL) } \\
\hline Median (IQR) & $611(64-332500)$ & $\begin{array}{l}57400(11300- \\
2180000)\end{array}$ & $549(20-245)$ & $\begin{array}{l}1230(130- \\
143000)\end{array}$ & $<0.05^{*}$ \\
\hline$<1000, n / N$ & - & $3 / 19(15.8)$ & $24 / 26(92.3)$ & $16 / 35(45.7)$ & $<0.05^{*}$ \\
\hline $1000-10000, n / N$ & - & $0 / 19(0)$ & $2 / 26(7.7)$ & $9 / 35(25.7)$ & \\
\hline$\geq 10000, n / N$ & - & $16 / 19(84.2)$ & $0 / 26(0)$ & $10 / 35(28.6)$ & \\
\hline Time on ART (days), median (IQR) & - & - & $85(49-355)$ & $168(15-354)$ & 0.373 \\
\hline \multicolumn{6}{|l|}{ TB regimen at presentation, $n / N(\%)$} \\
\hline $\mathrm{RH}$ & $13 / 100(13.0)$ & $3 / 46(6.5)$ & - & $10 / 54(18.5)$ & 0.085 \\
\hline RHZE & $73 / 100(73.0)$ & $35 / 46(76.1)$ & - & $38 / 54(70.4)$ & \\
\hline MDR & $2 / 100(2.0)$ & $0 / 46(0)$ & - & $2 / 54(3.7)$ & \\
\hline Other & $12 / 100(12.0)$ & $8 / 46(17.4)$ & - & $4 / 54(7.4)$ & \\
\hline \multicolumn{6}{|l|}{ TB diagnosis, $n / N(\%)$} \\
\hline Confirmed & $76 / 100(76.0)$ & $38 / 46(82.6)$ & - & $38 / 54(70.4)$ & 0.473 \\
\hline Empirical & $23 / 100(23.0)$ & $8 / 46(17.4)$ & - & $15 / 54(27.7)$ & \\
\hline Presumptive & $1 / 100(1.0)$ & $0 / 46(0)$ & - & $1 / 54(1.9)$ & \\
\hline \multicolumn{6}{|l|}{ Site of TB, $n / N(\%)$} \\
\hline Pulmonary & $55 / 98(56.1)$ & $21 / 45(46.7)$ & - & $34 / 53(64.2)$ & 0.343 \\
\hline Disseminated & $35 / 98(35.7)$ & $20 / 45(44.5)$ & - & $15 / 53(28.3)$ & \\
\hline Extrapulmonary & $4 / 98(4.1)$ & $2 / 45(4.4)$ & - & $2 / 53(3.8)$ & \\
\hline Other & $4 / 98(4.1)$ & $2 / 45(4.4)$ & - & $2 / 53(3.8)$ & \\
\hline Time on TB treatment (days), median (IQR) & $19.5(5.0-59.5)$ & $14.0(0.0-35.0)$ & - & $41.0(15.0-92.5)$ & $0.02^{*}$ \\
\hline
\end{tabular}

Continued ... 
Table 1. (continued) Characteristics of patients admitted for DILI between October 2015 and February 2017

\begin{tabular}{|c|c|c|c|c|c|}
\hline & All $(N=129)$ & $\begin{array}{l}\text { TB DILI } \\
(N=46)^{\dagger}\end{array}$ & $\begin{array}{l}\text { ART DILI } \\
(N=29)^{*}\end{array}$ & $\begin{array}{l}\text { Mixed DILI } \\
(N=54)^{\varsigma}\end{array}$ & $p$-value \\
\hline \multicolumn{6}{|l|}{ HBsAg, $n / N(\%)$} \\
\hline Negative & $111 / 129(86.1)$ & $38 / 46(82.6)$ & $26 / 29(29.7)$ & $47 / 54(87.0)$ & \multirow[t]{3}{*}{0.681} \\
\hline Positive & $6 / 129(4.7)$ & $2 / 46(4.4)$ & $2 / 29(6.9)$ & $2 / 54(3.7)$ & \\
\hline Not done & $12 / 129(9.3)$ & $6 / 46(13.0)$ & $1 / 29(3.5)$ & $5 / 54(9.3)$ & \\
\hline \multicolumn{6}{|l|}{ Hep C ab, $n / N(\%)$} \\
\hline Negative & $102 / 129(79.1)$ & $34 / 46(73.9)$ & $26(89.7)$ & $42 / 54(77.8)$ & \multirow[t]{3}{*}{0.252} \\
\hline Positive & $0 / 129(0)$ & $0 / 46(0)$ & $0 / 29(0)$ & $0 / 54(0)$ & \\
\hline Not done & $27 / 129(20.9)$ & $12 / 46(26.1)$ & $3 / 29(10.3)$ & $12 / 54(22.2)$ & \\
\hline \multicolumn{6}{|l|}{ Liver function, median (IQR) } \\
\hline $\operatorname{ALT}(\mathrm{U} / \mathrm{L})$ & $193(79-438)$ & $135(79-299)$ & $626(292-1293)$ & $125(45-291)$ & $<0.05^{*}$ \\
\hline Total bilirubin (mmol/L) & $75(25-153)$ & $60(17-108)$ & $193(75-286)$ & $64(21-127)$ & $<0.05^{\star}$ \\
\hline GGT (U/L) & $245(135-449)$ & $200(122-304)$ & $322(131-519)$ & $294(172-497)$ & $0.041^{*}$ \\
\hline $\operatorname{ALP}(\mathrm{U} / \mathrm{L})$ & $221(133-362)$ & $192(100-342)$ & $272(177-342)$ & $254(133-465)$ & 0.274 \\
\hline Albumin $(\mathrm{g} / \mathrm{L})$ & $20(17-26)$ & $17(16-22)$ & $30(25-33)$ & $19(15-24)$ & $<0.05^{*}$ \\
\hline INR & $1.34(1.2-1.8)$ & $1.31(1.17-1.58)$ & $1.39(1.21-2.15)$ & $1.35(1.20-1.81)$ & 0.241 \\
\hline \multicolumn{6}{|l|}{ Severity of DILI, $n / N(\%)$} \\
\hline Mild & $21 / 127(16.5)$ & $8 / 44(18.2)$ & $0 / 29(0)$ & $13 / 54(24.1)$ & \multirow[t]{3}{*}{0.056} \\
\hline Moderate & $35 / 127(27.6)$ & $13 / 44(29.6)$ & $11 / 29(37.9)$ & $11 / 54(20.4)$ & \\
\hline Severe & $71 / 127(55.9)$ & $23 / 44(52.3)$ & $18 / 29(62.1)$ & $30 / 54(55.6)$ & \\
\hline \multicolumn{6}{|c|}{ Pattern of liver injury (R value), $n / N(\%)$} \\
\hline Hepatocellular & $40 / 129(31.0)$ & $12 / 46(26.1)$ & $17 / 29(58.6)$ & $11 / 54(20.4)$ & \multirow[t]{3}{*}{$0.0010^{*}$} \\
\hline Cholestatic & $55 / 129(42.6)$ & $20 / 46(43.5)$ & $4 / 29(13.8)$ & $31 / 54(57.4)$ & \\
\hline Mixed & $34 / 129(26.4)$ & $14 / 46(30.4)$ & $8 / 29(27.6)$ & $12 / 54(22.2)$ & \\
\hline \multicolumn{6}{|c|}{ 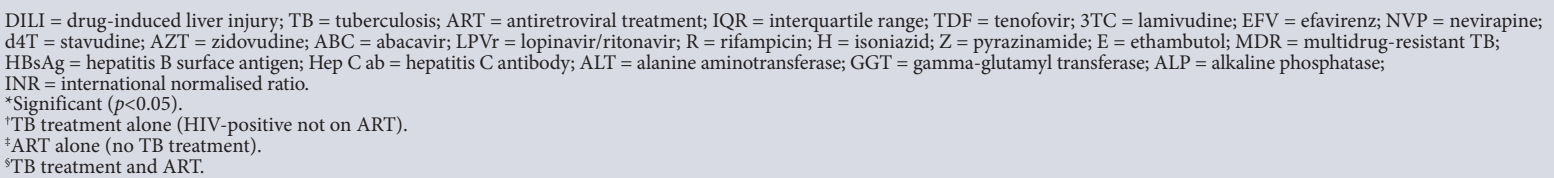 } \\
\hline
\end{tabular}

$193(75$ - 286) $\mathrm{mmol} / \mathrm{L}$ and $322(131$ - 519) $\mathrm{IU} / \mathrm{L}$, respectively. Hepatocellular injury therefore predominated in those with ART DILI (58.6\%), while cholestatic injury was noted more in those with mixed DILI (57.4\%).

\section{Patients on anti-TB therapy}

On admission, 100 patients were on anti-TB therapy. The majority $(n=73)$ were on rifampicin, isoniazid, pyrazinamide and ethambutol (RHZE), and 13 patients were on rifampicin and isoniazid only. Among those on anti-TB therapy, $76.0 \%$ had a confirmed diagnosis and more than half (56.1\%) had pulmonary TB. Of TB patients with DILI, 46 presented with TB DILI and 54 with mixed DILI. For those with TB DILI, the median (IQR) CD4 count was 37.5 (13 - 70) cells/ $\mu \mathrm{L}$ and $84.2 \%$ had a viral load $\geq 10000$ copies $/ \mathrm{mL}$. The median time between TB treatment initiation and DILI was 14 days for patients with TB DILI v. 41 days in those with mixed DILI.

The median (IQR) ALT, total bilirubin and GGT in patients with TB DILI was 135 (79 - 299) IU/L, 60 (17 - 108) mmol/L and 200 (122 - 304) IU/L, respectively. Just over half (52.3\%) of those with TB DILI were classified as having severe disease, with the main pattern of liver injury being cholestatic (43.5\%).

\section{Patient outcomes}

Of patients with ART DILI, only $7.4 \%$ were discharged on an FDC regimen, whereas $92.6 \%$ required a modified regimen (in which efavirenz was substituted with a protease inhibitor such as lopinavir-ritonavir) (Table 2).
Only 6 patients with TB DILI were discharged on the first-line regimen (RHZE) after successful re-challenge, and another 6 patients on a regimen containing rifampicin and isoniazid only. The majority of the patients $(66.7 \%)$ were discharged on a modified regimen containing only one first-line drug or a bridging therapy regimen (such as moxifloxacin and ethambutol). Of those with mixed DILI, only 6 (13.3\%) were discharged on RHZE, and 24 (57.5\%) required a modified ART regimen. Patients were followed from admission until the earliest of death $(n=21 ; 10$ with TB DILI, 2 with ART DILI and 9 with mixed DILI) or discharge (after a median (IQR) of 14.0 (9 - 23) days).

Table 3 shows the results of ultrasound examination and the association with severity of DILI or as predictors of all-cause mortality. Presenting with an enlarged liver $(>15 \mathrm{~cm})$ as opposed to a liver of normal size was associated with severe DILI at admission (RR 1.39; 95\% CI 0.99 - 1.96; $p=0.057$ ). In addition, patients who presented with an echogenic liver as opposed to a normal liver were five times more likely to die (HR 4.98; 95\% CI 1.39 - 17.85; $p=0.014$ ). We found no association between use of concomitant hepatotoxic medication (e.g. fluconazole, co-trimoxazole, amoxicillin-clavulanic acid, paracetamol) or herbal medication and the severity of DILI at admission. Concomitant use of herbal or prescription medication did not predict all-cause mortality (Table 3 ).

Deaths were similar across sexes (male v. female: adjusted hazard ratio (aHR) $0.79 ; 95 \%$ CI $0.32-1.93$ ), ages ( $\geq 35$ v. $<35$ years: aHR 0.94; 95\% CI $0.39-2.27$ ) and pattern of liver injury (Table 4 ). Type of DILI (ART, TB or mixed) was not a predictor of all- 
Table 2. Outcomes of patients admitted for DILI between October 2015 and February 2017

\begin{tabular}{|c|c|c|c|c|c|}
\hline & All $(N=129)$ & TB DILI $(N=46)^{\dagger}$ & ART DILI $(N=29)^{*}$ & Mixed DILI $(N=54)^{5}$ & $p$-value \\
\hline \multicolumn{6}{|l|}{ Outcome, $n / N(\%)$} \\
\hline Discharge & $108 / 129(83.7)$ & $36 / 46(78.3)$ & $27 / 29(93.1)$ & $45 / 54(83.3)$ & 0.263 \\
\hline Died & $21 / 129(16.3)$ & $10 / 46(21.7)$ & $2 / 29(6.9)$ & $9 / 54(16.7)$ & \\
\hline \multirow[t]{2}{*}{ Follow-up time (days), median (IQR) } & $14.0(9.0-23.0)$ & $19.0(12.0-35.0)$ & $9.0(6.0-13.0)$ & $15.0(10.0-23.0)$ & $<0.05^{\star}$ \\
\hline & All $(N=108)$ & TB DILI $(N=36)^{\dagger}$ & ART DILI $(N=27)^{\ddagger}$ & ART/TB DILI $(N=45)^{\S}$ & \\
\hline \multicolumn{6}{|l|}{ ART regimen at discharge, $n / N(\%)$} \\
\hline Same as regimen at presentation & $22 / 72(30.6)$ & - & $2 / 27(7.4)$ & $20 / 45(44.4)$ & $0.010^{*}$ \\
\hline Modified & $50 / 72(69.4)$ & - & $25 / 27(92.6)$ & $25 / 45(55.6)$ & \\
\hline \multicolumn{6}{|l|}{ TB regimen at discharge, $n / N(\%)$} \\
\hline Same as regimen at presentation & $20 / 81(24.7)$ & $11 / 36(30.6)$ & & $9 / 45(20.0)$ & 0.274 \\
\hline Modified & $61 / 81(75.3)$ & $25 / 36(69.4)$ & & $36 / 45(80.0)$ & \\
\hline \multicolumn{6}{|l|}{ TB regimen at discharge, $n / N(\%)$} \\
\hline $\mathrm{RH}$ & $19 / 81(23.5)$ & $6 / 36(16.7)$ & - & $13 / 45(28.9)$ & 0.453 \\
\hline RHZE & $12 / 81(14.8)$ & $6 / 36(16.7)$ & - & $6 / 45(13.3)$ & \\
\hline MDR & $32 / 81(39.5)$ & $17 / 36(47.2)$ & - & $15 / 45(33.3)$ & \\
\hline Other & $18 / 81(19.8)$ & $7 / 36(19.4)$ & - & $11 / 45(24.2)$ & \\
\hline \multicolumn{6}{|c|}{$\begin{array}{l}\mathrm{DILI}=\text { drug-induced liver injury; } \mathrm{TB}=\text { tuberculosis; } \mathrm{ART}=\text { antiretroviral treatment; } \mathrm{IQR}=\text { interquartile range; } \mathrm{R}=\text { rifampicin; } \mathrm{H}=\text { isoniazid; } \mathrm{Z}=\text { pyrazinamide; } \mathrm{E}=\text { ethambutol; } \\
\mathrm{MDR}=\text { multidrug-resistant } \mathrm{TB} \text {. } \\
\text { `Significant }(p<0.05) \text {. } \\
\text { 'TB treatment alone }(\mathrm{HIV} \text {-positive not on ART). } \\
\text { ₹ART alone (no TB treatment). } \\
\text { } \text { TB treatment and ART. }\end{array}$} \\
\hline
\end{tabular}

cause mortality. In adjusted analysis, only severe DILI at admission predicted all-cause mortality (aHR 8.58; 95\% CI 1.13 - 65.4) (Table 4).

To account for differences in follow-up time by severity of DILI (i.e. maximum 83 days for mild, 113 days for moderate and 120 days for severe), we conducted a sensitivity analysis. Fig. 1 shows the probability of retention in care over 60 days by severity of DILI. By 60 days, compared with patients admitted with mild DILI, those with severe DILI had a significantly elevated risk of death (aHR 11.3; 95\% CI 1.46 - 87.54).

\section{Discussion}

Among HIV-positive patients admitted with DILI, we found that $24.7 \%$ and $30.6 \%$ of patients could be re-challenged on standard ART or anti-TB regimens, respectively, at discharge. Mortality was high, although similar to what has been described for clinical cohorts in the same setting (9\% among HIVpositive patients within the first 6 months of ART, 23\% among HIV-positive patients on ART with prevalent TB). ${ }^{[9,10]}$

Consistent with other studies, we found that among HIV-positive adults admitted for DILI, most patients had a mixed DILI following combination ART and anti-TB therapy ${ }^{[11,12]}$ Those with TB DILI presented a median of 14 days after drug initiation, or 41 days if they were on anti-TB therapy and ART, whereas in another study among outpatients presenting with TB DILI, the average time between TB treatment and time to DILI

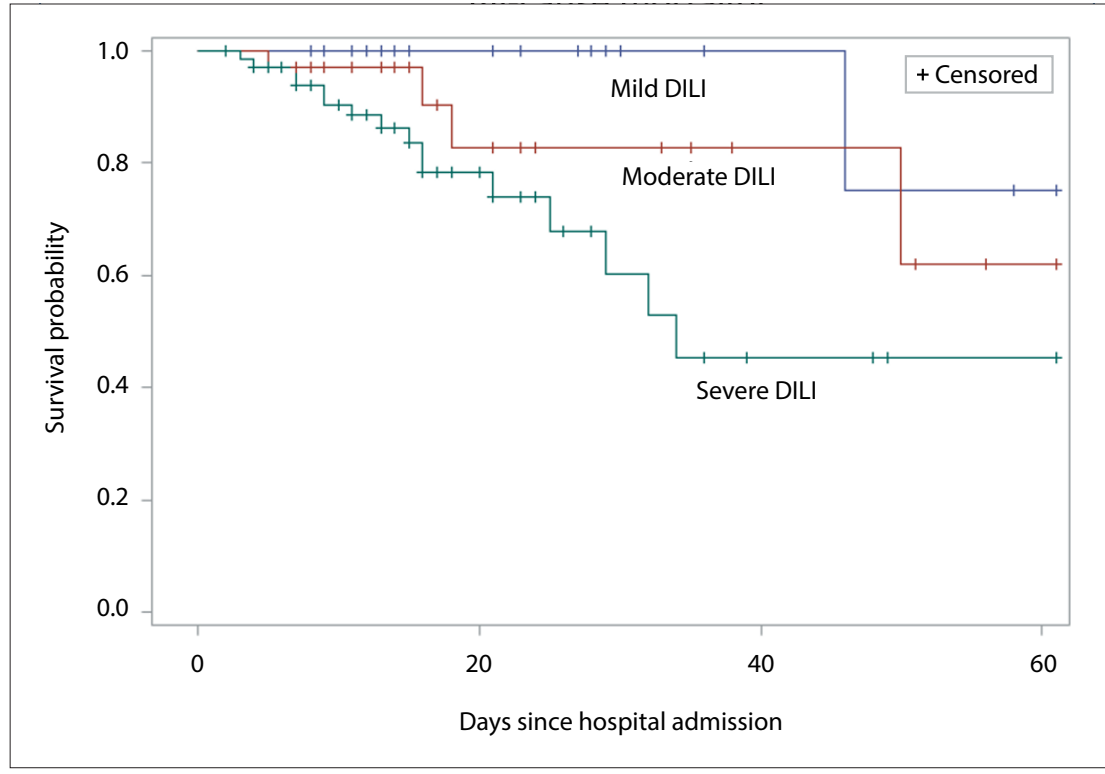

Fig. 1. Kaplan-Meier survival estimates for all-cause mortality following hospital admission $(\mathrm{n}=21)$. (DILI $=$ drug-induced liver injury. $)$

diagnosis was 31 days. ${ }^{[12]}$ This difference reflects the severity of DILI requiring hospitalisation. The longest time between treatment initiation and presentation with DILI in this group was 92 days.

Patients with ART DILI presented later, with some presenting as late as 355 days after initiation of ART. Even though patients with ART DILI presented later with some immune reconstitution (higher CD4 counts and lower viral loads than those with TB DILI or mixed DILI), this group presented with greater severity of DILI and a predominantly hepatocellular pattern. Jaundice, seen in nearly three-quarters of our patients, along with elevated liver function tests, despite responding to ART, should alert clinicians to the possibility of ART DILI. Despite the late presentation and greater severity of DILI, patients with ART DILI had the shortest hospital stay and the lowest mortality rate. While an FDC regimen is considered to pose a low risk for development of hepatotoxicity, $<10 \%$ of patients with ART DILI could be successfully re-challenged back to the original regimen at discharge. Management 


\begin{tabular}{|c|c|c|c|c|}
\hline & Severe DILI $(N=71), n / N(\%)$ & Crude RR (95\%) CI & Death $(N=21), \mathrm{n} / N(\%)$ & Crude HR (95\% CI) \\
\hline \multicolumn{5}{|c|}{ Concomitant medication } \\
\hline \multicolumn{5}{|c|}{ Bactrim } \\
\hline No & $57 / 98(58.2)$ & 1.0 & $16 / 98(16.3)$ & 1.0 \\
\hline Yes & $13 / 25(52.0)$ & $0.89(0.59-1.35)$ & $4 / 25(16.0)$ & $0.95(0.32-2.85)$ \\
\hline \multicolumn{5}{|c|}{ Fluconazole } \\
\hline No & $66 / 114(57.9)$ & 1.0 & 19/114 (16.7) & 1.0 \\
\hline Yes & $4 / 9(44.4)$ & $0.77(0.36-1.62)$ & $1 / 9(11.1)$ & $0.37(0.05-2.98)$ \\
\hline \multicolumn{5}{|c|}{ Paracetamol } \\
\hline No & 69/122(56.6) & - & 20/122(16.4) & \\
\hline Yes & $1 / 1(100)$ & - & $0 / 1(0)$ & \\
\hline \multicolumn{5}{|c|}{ Amox-clavulanate } \\
\hline No & 65/111 (58.6) & 1.0 & 16/111 (14.4) & 1.0 \\
\hline Yes & $5 / 12(41.7)$ & $0.71(0.36-1.41)$ & $4 / 12(33.3)$ & $1.88(0.62-5.70)$ \\
\hline \multicolumn{5}{|c|}{ Herbal medication } \\
\hline No & $70 / 123(56.9)$ & - & 20/123 (16.3) & - \\
\hline Yes & & - & & - \\
\hline \multicolumn{5}{|c|}{ Ultrasound results } \\
\hline \multicolumn{5}{|c|}{ Enlarged liver $(\geq 15 \mathrm{~cm})$} \\
\hline No & $31 / 54(57.4)$ & 1.0 & $8 / 54(14.8)$ & 1.0 \\
\hline Yes & $12 / 15(80.0)$ & $1.39(0.99-1.96)^{*}$ & $3 / 15(20.0)$ & $1.46(0.38-5.62)$ \\
\hline \multicolumn{5}{|c|}{ Echogenic } \\
\hline No & $30 / 52(57.7)$ & 1.0 & $4 / 52(7.7)$ & 1.0 \\
\hline Yes & $13 / 17(76.5)$ & $1.33(0.94-1.88)$ & $7 / 17(41.2)$ & $4.98(1.39-17.85)^{*}$ \\
\hline \multicolumn{5}{|c|}{ Liver mass } \\
\hline No & $71 / 126(56.4)$ & - & 21/126 (16.7) & - \\
\hline Yes & $0 / 1(0)$ & - & $0 / 1(0)$ & - \\
\hline \multicolumn{5}{|c|}{ Micro-abscesses } \\
\hline No & $61 / 104(58.7)$ & 1.0 & 16/104 (15.4) & 1.0 \\
\hline Yes & $10 / 23(43.5)$ & $0.74(0.45-1.21)$ & $5 / 23(21.7)$ & $0.90(0.33-2.48)$ \\
\hline \multicolumn{5}{|c|}{ Intra-abdominal nodes } \\
\hline No & $33 / 49(67.4)$ & 1.0 & $7 / 49(14.3)$ & 1.0 \\
\hline Yes & $10 / 20(50.0)$ & $0.74(0.46-1.20)$ & $4 / 20(20.0)$ & $0.80(0.23-2.83)$ \\
\hline \multicolumn{5}{|l|}{ Ascites } \\
\hline No & $35 / 56(62.5)$ & 1.0 & $6 / 56(10.7)$ & 1.0 \\
\hline Yes & $8 / 13(61.5)$ & $0.98(0.61-1.58)$ & $5 / 13(38.5)$ & $2.26(0.68-7.58)$ \\
\hline \multicolumn{5}{|c|}{ Ultrasound evidence of $\mathrm{TB}$} \\
\hline No & $59 / 103(57.3)$ & 1.0 & $16 / 103(15.5)$ & 1.0 \\
\hline Yes & $12 / 24(50.0)$ & $0.87(0.57-1.35)$ & $5 / 24(20.8)$ & $0.87(0.32-2.40)$ \\
\hline
\end{tabular}

requires consideration of other causes of the deranged liver function, including viral hepatitis, other hepatotoxins, sepsis or a chronic liver condition. ${ }^{[12]}$

The features of DILI in HIV-positive patients hospitalised for TB DILI or mixed DILI were similar; both groups presented with low CD4 counts and high viral loads, were less likely to present with symptomatic DILI or to have deranged liver function tests than patients with ART DILI, and had a cholestatic liver function pattern. One in four patients with TB DILI or mixed DILI could be successfully re-challenged back to the original TB regimen at discharge, with a higher proportion (44.4\%) with mixed DILI re-challenged on their original ART regimen. In contrast to what we observed with ART DILI, while fewer patients with TB DILI or mixed DILI presented with severe DILI, these groups had the longest stay in hospital and a higher mortality rate $(21.7 \%$ and $16.7 \%$ v. $6.9 \%$ for ART DILI). Despite differences in presentation, only the severity of TB DILI at admission was a predictor of all-cause mortality. Age, gender, type of DILI or pattern of liver injury did not predict mortality. Revised monitoring algorithms with more frequent liver function tests (in the first 2 - 3 months of ART or TB treatment) may help to identify symptoms and raised liver enzymes early. Results suggest that patients with severe DILI and, while not significant, those with anti-TB or mixed DILI would probably benefit from early identification to reduce mortality.

\section{Study limitations}

The retrospective nature of the study has its limitations, including incomplete data on some patients, which limits interpretation of some results. Also, the study included patients admitted to a large urban tertiary hospital, so results may not be generalisable to other 
Table 4. Predictors of death among patients admitted for DILI between October 2015 and February $2017(N=129)$

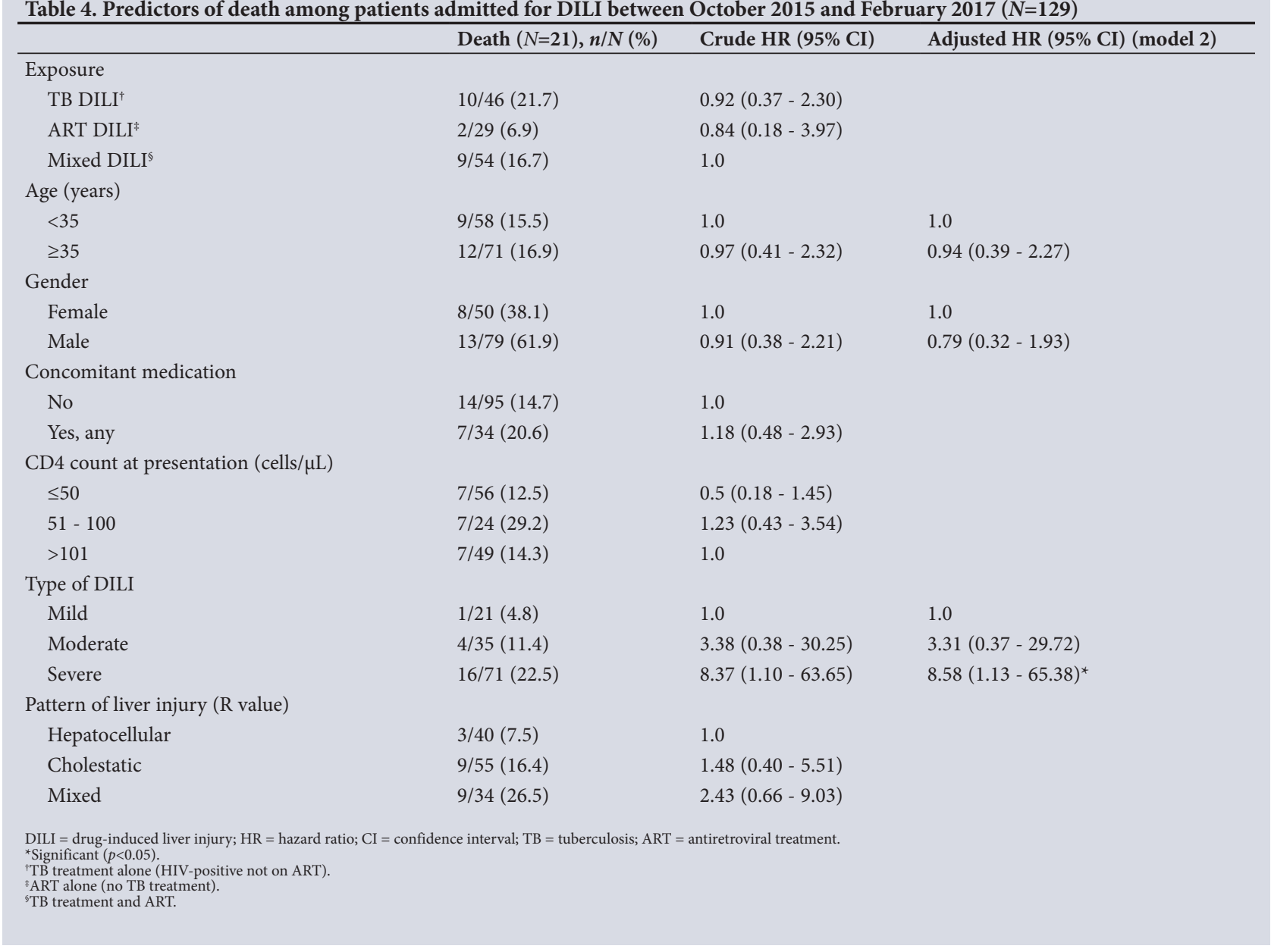

settings. We do not have any data on mortality after discharge, and it is possible that DILI could have recurred in some patients after re-challenge in an outpatient setting, potentially underestimating all-cause mortality associated with DILI. Data are not available to determine causes of death and to establish whether it was due to liver failure or sepsis as a result of drug withdrawal. Small numbers and a lack of data on duration or dose of concomitant herbal or prescription medication may have limited our ability to analyse whether these contribute to the severity of DILI, or place patients at increased risk for all-cause mortality. Similarly, only five patients had biopsy results, which limited our ability to consider this in the analysis. Prospective studies of DILI patients with longer followup would overcome some of these limitations with more complete data (e.g. including cause of death from mortuary registers). Future studies and/or analyses should also consider biopsy results for patient selection to rule out other causes of liver dysfunction if suspected.

Despite these limitations, we would like to make the following recommendations. Patients should be educated on the symptoms of DILI so that they can identify symptoms, distinguish these from adverse effects caused by drugs, and present earlier while still in the mild stage of illness. Patients should also be provided with a management plan in the event that DILI symptoms arise, and highrisk patients should be targeted for frequent monitoring of liver function (e.g. every 2 weeks in the intensive phase of TB treatment and monthly thereafter, or every month for the first 3 months in patients on ART and then at selected intervals thereafter).

\section{Conclusions}

This study is one of the few analyses of hospitalised patients with DILI in SA. Patients with TB DILI presented earlier, in the intensive phase of therapy, whereas those with ART DILI presented up to 1 year after ART initiation, highlighting the importance of maintaining a high index of suspicion and regular surveillance for earlier diagnosis of DILI. Among patients admitted with DILI, those with severe DILI were at increased risk of all-cause mortality. Large cohort studies with longer follow-up are required to predict which patients are at highest risk for DILI, the drugs responsible, and the long-term outcomes.

Declaration. The research for this study was done in partial fulfilment of the requirements for RM's MMed (Internal Medicine) degree at the University of the Witwatersrand.

Acknowledgements. None.

Author contributions. RM, PI and CNM conceived the study. RM collected the data. DE analysed the data. RM prepared the manuscript. PI, DE and CNM were responsible for critical revision of the manuscript. Funding. This study was made possible by the generous support of the American people and the President's Emergency Plan for AIDS Relief (PEPFAR) through the US Agency for International Development (USAID) under the terms of Cooperative Agreements AID674-A-12-00029 and 72067419CA00004 to the Health Economics and Epidemiology Research Office. The contents are the responsibility of the 
authors and do not necessarily reflect the views of PEPFAR, USAID or the US government. The funders had no role in the study design, collection, analysis and interpretation of the data, manuscript preparation or the decision to publish.

\section{Conflicts of interest. None.}

1. Statistics South Africa. Mid-year population estimates 2015. Statistical release P0302. Pretoria: Stats SA, 2015. https://www.statssa.gov.za/publications/P0302/P03022015.pdf (accessed 25 November 2020).

2. Hoffmann CJ, Charalambous S, Thio CL, et al. Hepatotoxicity in an African antiretroviral therapy cohort: The effect of tuberculosis and hepatitis B. AIDS 2007;21(10):1301-1308. https://doi. org/10.1097/QAD.0b013e32814e6b08

3. Jong E, Conradie F, Berhanu R, et al. Consensus statement: Management of drug-induced liver injury in HIV-positive patients treated for TB. South Afr J HIV Med 2013;14(3):113-119. https://doi. injury in HIV-positive patiens

4. Montessori V, Press N, Harris M, Akagi L, Montaner IS. Adverse effects of antiretroviral therapy for HIV infection. Can Med Assoc J 2004;170(2):229-238.

5. Schaberg T, Rebhan K, Lode H. Risk factors for side-effects of isoniazid, rifampin and pyrazinamide 5. Schaberg T, Rebhan K, Lode H. Risk factors for side-effects of isoniazid, rifampin and pyrazinamide
in patients hospitalised for pulmonary tuberculosis. Eur Respir J 1996;9(10):2026-2030. https://doi. org/10.1183/09031936.96.09102026
6. Nunez M. Hepatotoxicity of antiretrovirals: Incidence, mechanisms and management. J Hepatol 2006;44(1 Suppl):S132-S139. https://doi.org/10.1016/j.jhep.2005.11.027

7. Cohen K Meites G. Mangent Curr Opin HIV AIDS 2010;5(1):61-69. https://doi.org/10.1097/COH.0b013e3283339309

8. Berry KM, Rodriguez CA, Berhanu RH, et al. Treatment outcomes among children, adolescents, and 8. Berry KM, Rodriguez CA, Bercunu RH, et al. Treatment outcomes among children, adolescents, and
adults on treatment for tuberculosis in two metropolitan municipalities in Gauteng Province, South adults on treatment for tuberculosis in two metropolitan municipalities in Gauteng Province, Sou Africa. BMC Public Health 2019;19(1):973. https://doi.org/10.1186/s12889-019-7257-4

9. Westreich D, Fox MP, van Rie A, Maskew M. Prevalent tuberculosis and mortality among HAART initiators. AIDS 2012;26(6):770-773. https://doi.org/10.1097/QAD.0b013e328351f6b

0. Fox MP, Maskew M, MacPhail AP, et al. Cohort profile: The Themba Lethu clinical cohort, Johannesburg, South Africa. Int J Epidemiol 2013;42(2):430-439. https://doi.org/10.1093/ije/dys029

11. Yimer G, Aderaye G, Amogne W, et al. Anti-tuberculosis therapy-induced hepatotoxicity among Ethiopian HIV-positive and negative patients. PloS ONE 2008;3(3):e1809. https://doi.org/10.1371/ journal.pone.0001809

12. Naidoo S, Evans D, Jong E, Mellet K, Berhanu R. Outcomes of TB/HIV co-infected patients presenting with antituberculosis drug-induced liver injury. S Afr Med J 2015;105(5):393-396. https://doi. org/10.7196/SAMJ.8217

Accepted 2 December 2020 